\title{
Plasma lipidomics are associated with insulin sensitivity and secretion in overweight and obese non-diabetic adults
}

Aya Mousa $^{1}$, Negar Naderpoor ${ }^{1,2}$, Estifanos Baye ${ }^{1}$, Josphin Johnson ${ }^{1}$, Natalie Mellett ${ }^{3}$, Peter Meikle ${ }^{3}$, Mosearch and Implementation Barbora de Courten ${ }^{1,2}$

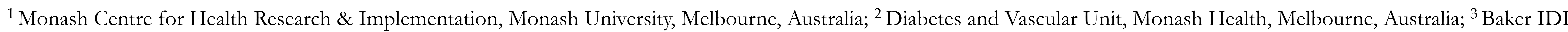
Heart and Diabetes Institute, Melbourne, Australia. Correspondence: barbora.decourten@monash.edu

\section{Background}

- Dyslipidaemia is a major risk factor for type 2 diabetes and cardiovascular disease.

- Lipidomics is a novel method providing new insights into the role of lipid metabolism in the pathophysiology of these diseases.

- However, human studies examining lipidomics and glucose metabolism are limited, and no previous studies have examined relationships between lipidomics and insulin secretion in humans.

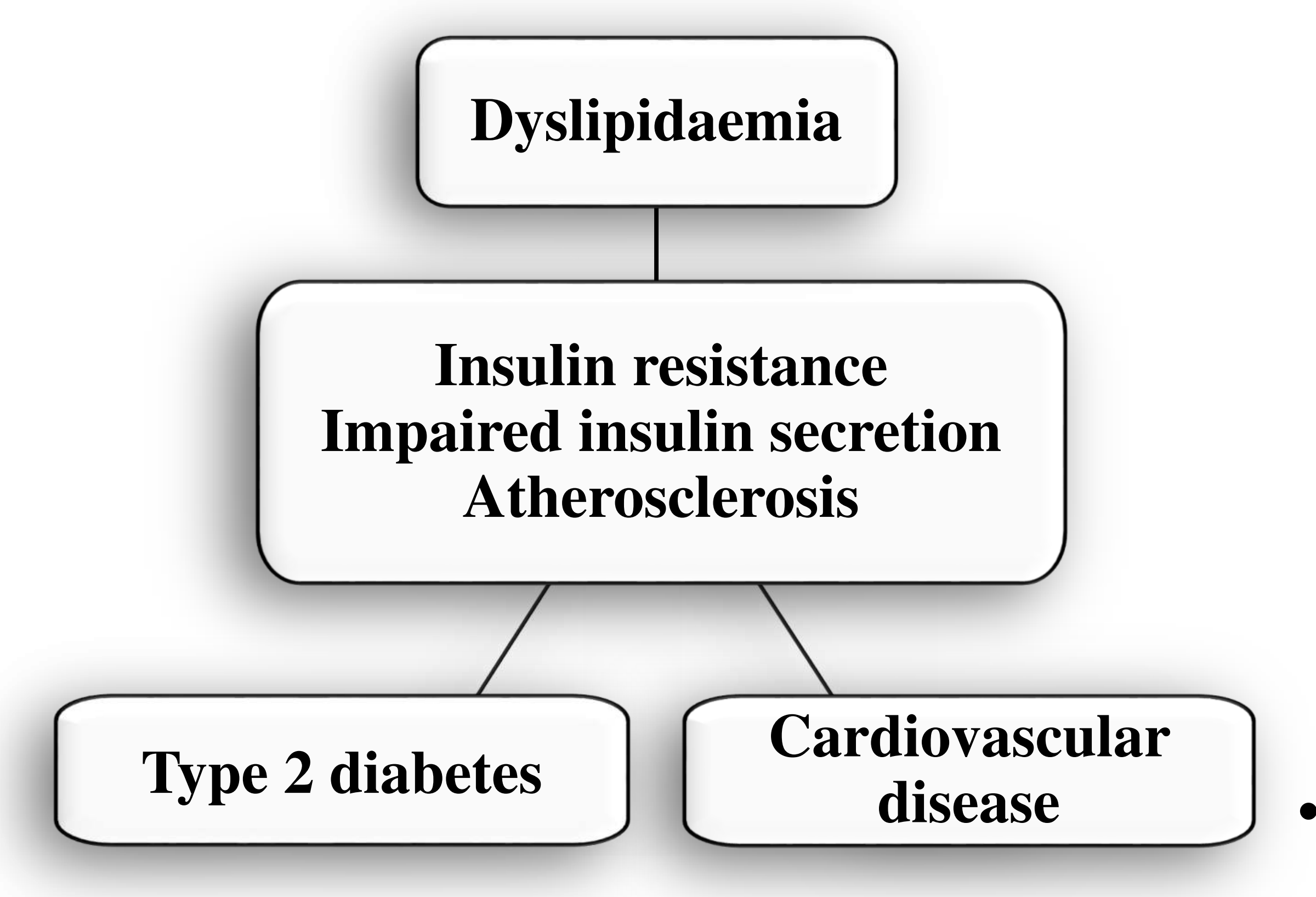

\section{Hypothesis}

We hypothesized that dysregulation of certain lipid species or classes will be associated with reduced insulin sensitivity and impaired insulin secretion.

\section{$\underline{\text { Aims }}$}

To examine whether certain lipid species/ classes are associated with insulin sensitivity and/or secretion measured by goldstandard methods, as well as other cardiometabolic risk factors in overweight or obese non-diabetic adults.

\section{Study population}

- 65 participants (41M / 24F):

- healthy, non-diabetic adults (WHO guidelines)

- age between 18 and $60 \mathrm{yrs}$

- overweight or obese (body mass index $[\mathrm{BMI}] \geq 25 \mathrm{~kg} / \mathrm{m}^{2}$ )

- non-smokers and not taking medications or using illicit drugs

- no chronic diseases or clinical or laboratory signs of acute or chronic inflammation

- not pregnant, lactating, or peri-/post-menopausal

\section{Methods}

- Anthropometry: BMI and \% body fat (DEXA)

- Glucose metabolism: insulin sensitivity (hyperinsulinemic euglycemic clamps); insulin secretion (intravenous glucose tolerance tests); glucose tolerance (oral glucose tolerance tests).

- Serum inflammatory markers: high sensitivity C-reactive protein (hsCRP; immunoturbidimetry); tumor necrosis factor (TNF), interleukins (IL)-1 $\beta, 6,10$, and adipokines: leptin, resistin, adiponectin, and adipsin (multiplex).

- Lipidomics: liquid chromatography electrospray mass spectrometry: 459 lipid species across 26 lipid classes.

- Statistical analyses: Matlab software. All analyses adjusted for multiple testing using Benjamini Hochberg (BH) correction.

\section{Sample characteristics}

\begin{tabular}{lc}
\hline & Mean \pm SD or $\mathbf{~}$ \\
\hline Male/ Female, $n$ & $41 / 24$ \\
Age (years) & $31.3 \pm 8.5$ \\
BMI $\left(\mathrm{kg} / \mathrm{m}^{2}\right)$ & $31.5 \pm 5.3$ \\
Body fat $(\%)$ & $40.1 \pm 8.7$ \\
\hline
\end{tabular}

\section{Results: Glucose metabolism}

In univariable analysis, insulin sensitivity was not associated with lipid species or classes. However, after adjustment for age, sex, and body fat, insulin sensitivity was inversely associated with the dhCer lipid class and several DG and TG lipid species ( $\mathrm{p}<0.05$; Fig 1 ).

- Total and second phase insulin secretion were positively associated with the LPI lipid class (Fig 2), which remained significant after adjusting for age, sex and body fat (both $\mathrm{p}<0.02$ ).

- Fasting and 2-hour glucose were not associated with any lipid classes/species (all p>0.05).

Fig 1. Insulin sensitivity is associated with the dhCer class $\&$ DG \& TG species after adjustment for age, sex \& body fat

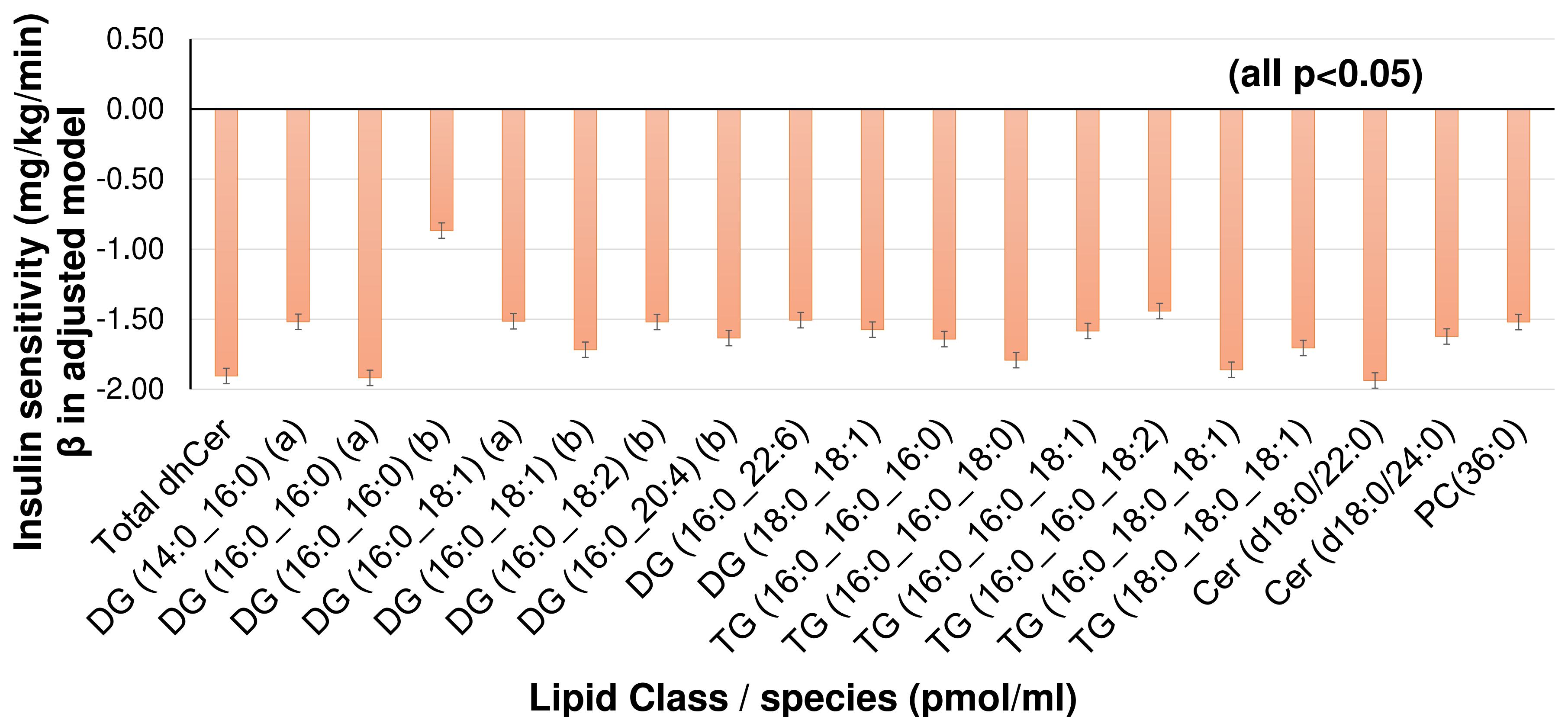

Fig 2. Insulin secretion is associated with the LPI class
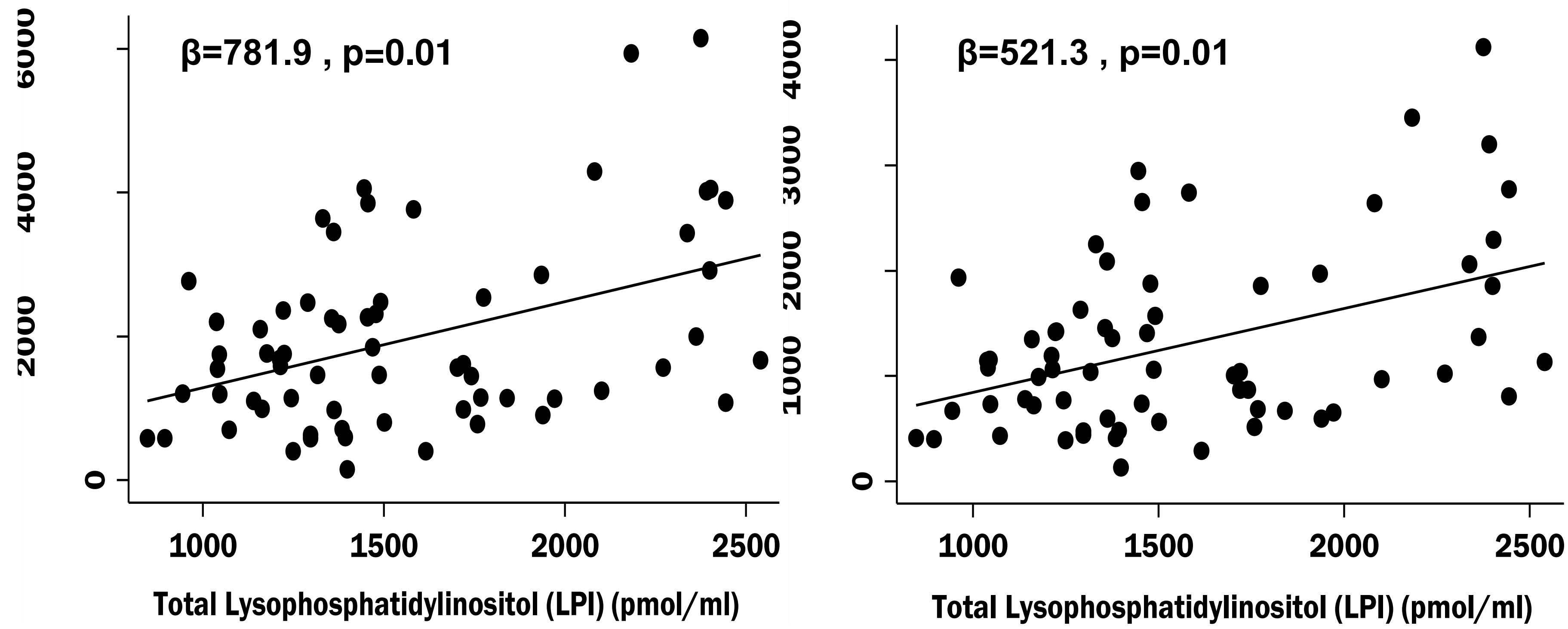

\section{Results: Inflammation}

- IL-1 $\beta$ was positively associated with the CE lipid class (Fig 3); however this was attenuated after adjustment for age, sex, and body fat $(\mathrm{p}=0.1)$.

- Adiponectin was inversely associated with several DG and TG lipid species (Fig 4), but this was attenuated after adjustment for age, sex, and body fat (all $\mathrm{p}>0.07$ ).

Fig 3. IL1 $\beta$ is associated with the CE class

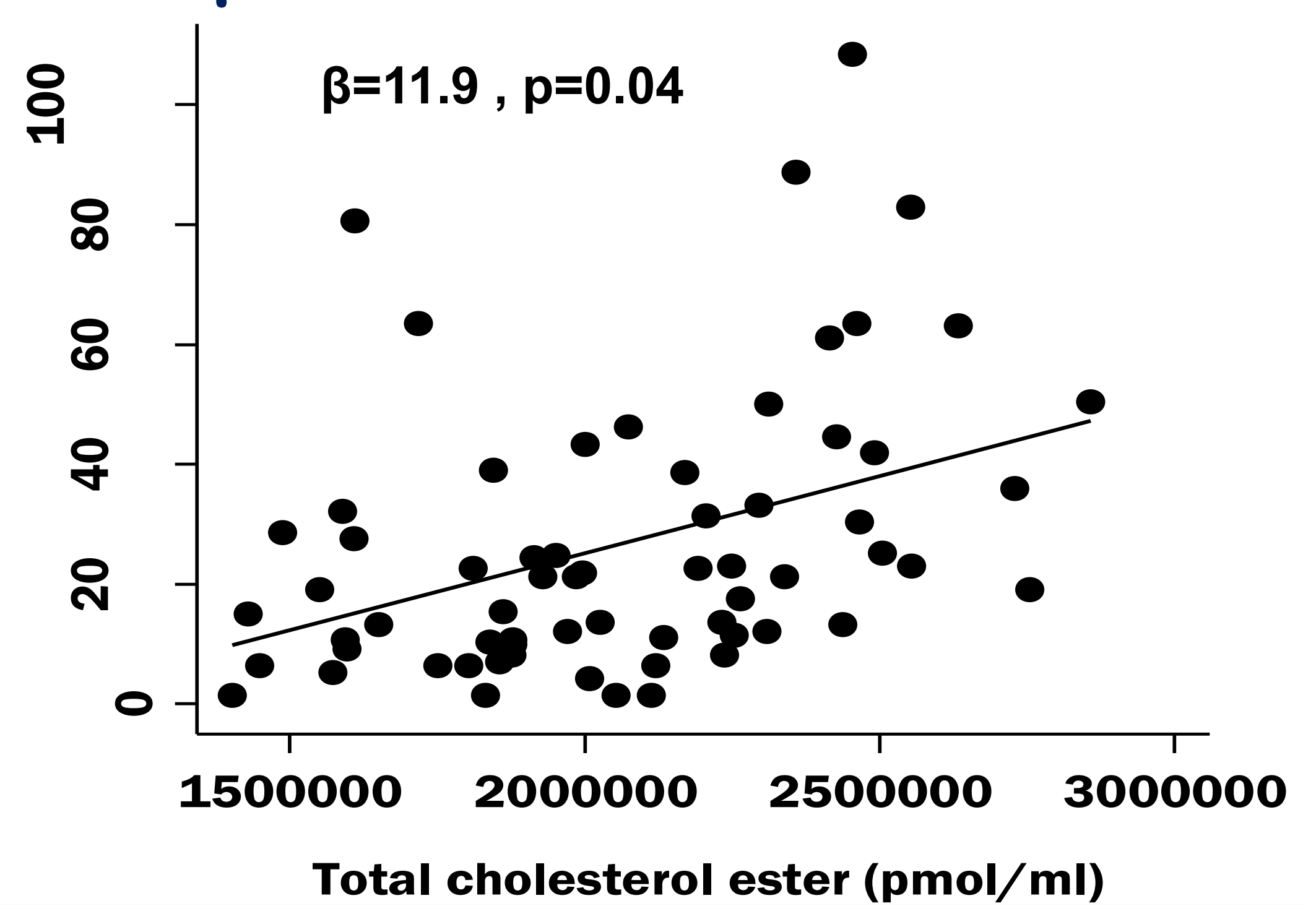

Fig 4. Adiponectin is associated with DG and TG lipid species

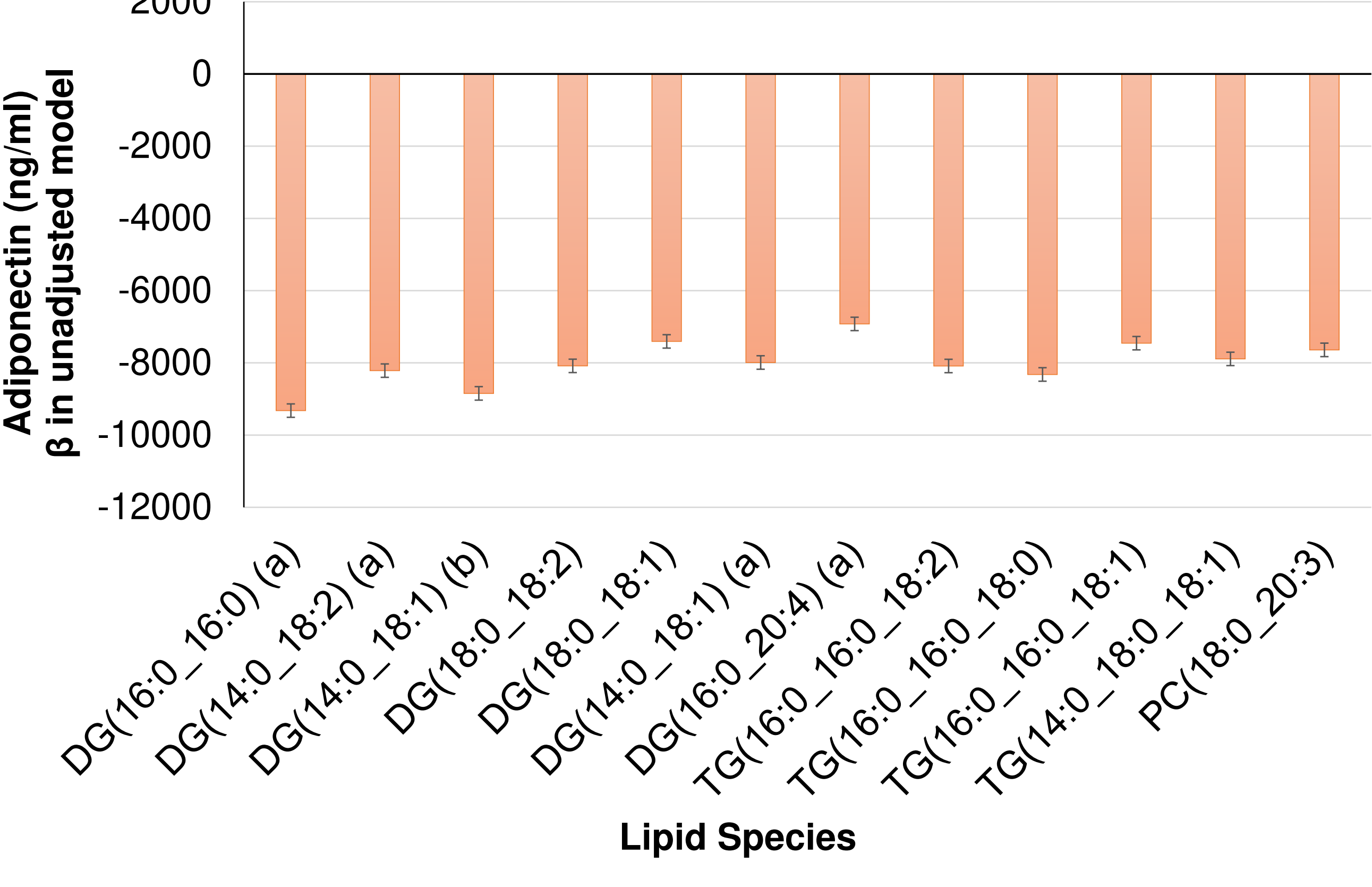

- There were no associations between lipid classes/species and hsCRP, TNF, IL-6 or IL-10, as well as with leptin, resistin, and adipsin (all $\mathrm{p}>0.05$ after $\mathrm{BH}$ correction).

\section{Conclusions}

- Our findings for insulin sensitivity and inflammation are consistent with previous studies implicating sphingolipids (dhCer) and glycerolipids (DG and TG) in insulin resistance and chronic inflammation.

- This is the first study examining the relationships between lipidomics and insulin secretion in humans.

- We report the novel finding that insulin secretion is positively associated with the LPI lipid class, which is consistent with mouse models showing that LPI has insulinotropic effects.

\section{$\underline{\text { References }}$}

-Weir, et al. J Lipid Res, 2013

- Tonks et al., Obesity, 2016

Pietiläinen, et al., PlosOne, 2007

\section{Abbreviations}

LPI: lysophosphatidylinositol; DG: diacylglycerol; TG; triacylglycerol; Cer: ceramides; dhCer: dihydroceramides; $\mathbf{C E}$ : cholesterol ester; PC: phosphatidylcholine. 